\title{
Two-Photon Responsive Porphyrinic Metal-Organic Framework Involving Fenton-Like Reaction for Enhanced Photodynamic and Sonodynamic Therapy
}

\section{Wenyao Duan}

Anhui University

Bo Li

Anhui University

Wen Zhang

Anhui University

Jiaqi Li

Anhui University

Xin Yao

Anhui University

Yupeng Tian

Anhui University

Jun Zheng

Anhui University

Dandan Li ( $\square$ chemlidd@163.com )

Anhui University https://orcid.org/0000-0002-3504-8739

\section{Research Article}

Keywords: Metal-organic framework, Two-photon, Hypoxia, Sonodynamic therapy, Photodynamic therapy.

Posted Date: February 21st, 2022

DOI: https://doi.org/10.21203/rs.3.rs-1319887/v1

License: (1) (1) This work is licensed under a Creative Commons Attribution 4.0 International License.

Read Full License 


\section{Abstract}

Designing new oxygenation nanomaterials by oxygen-generating or oxygen-carrying strategies in hypoxia-associated anti-tumor therapy is a high priority target yet challenge. In this work, we fabricated a nanoplatform involving Fenton-like reaction, Pd@MOF-525@HA, to relieve tumor hypoxia via oxygengenerating strategy for enhanced oxygen-dependent anti-tumor therapy. Thereinto, the porphyrinic MOF525 can produce singlet oxygen $\left({ }^{1} \mathrm{O}_{2}\right)$ via light or ultrasonic irradiation for photodynamic and sonodynamic therapy. Notably, the well-dispersed Pd nanocubes within MOF-525 can convert $\mathrm{H}_{2} \mathrm{O}_{2}$ into $\mathrm{O}_{2}$ to mitigate the hypoxic environment for enhanced therapy outcome. Moreover, the two-photon activity and cancer cell specific targeting capability of Pd@MOF-525@HA gave rise to deeper tissue penetration and near-infrared light-induced fluorescence imaging to achieve precise guidance for cancer therapy. This work provides a feasible way in designing new oxygenation nanomaterials to relieve tumor hypoxia for enhanced cancer treatment.

\section{Introduction}

As the second leading cause of human death, cancer poses a grievous threat to human health $[1,2]$. Facing the characteristics of infinite proliferation and easy metastasis of tumor cells, traditional radiotherapy and chemotherapy in combating cancer cells show unsatisfactory treatment results due to the multidrug resistance and severe side effects [3-5]. In this sense, researchers have made great efforts to develop more effective treatment methods, such as sonodynamic therapy (SDT) and photodynamic therapy (PDT), which are noninvasive tools with high selectivity for local cancers given the generated reactive oxygen species (ROS) from the photo/sonosensitizers by laser/ultrasound irradiation in an aerobic environment [6-8]. Unfortunately, the hypoxic environment of the tumor significantly limits their efficiencies [9-12]. To this end, it is imperative to develop a valid PDT/SDT system that can relieve tumor hypoxia.

Presently, the PDT agents used in clinic are mainly porphyrin derivatives, the excitation wavelength of which are located in the visible region $(400-700 \mathrm{~nm}$ ) leads to poor penetration limiting their further application $[13,14]$. However, it is worth mentioning that these porphyrin derivatives can be employed as sonosensitizers to effectively compensate for the barrier of shallow penetration depth in PDT $[15,16]$. Notably, the above two cancer treatment methods are highly oxygen dependent, the severe hypoxic environment of tumor greatly limits the corresponding outcome [17-20]. To face this grand challenge, various solutions have been developed to fabricate new oxygenation nanomaterials [21-23]. Among of them, the introduction of oxygen-generating units triggered by Fenton/Fenton-like reaction was recognized to be one of the most promising approach [24-26]. Therefore, the search for platforms involving Fenton/Fenton-like reaction based on porphyrin derivatives, which can relieve tumor hypoxia via oxygen-generating strategy, to achieve enhanced therapy outcome is a extraordinary desired target.

Based on the above considerations, we fabricated a porphyrinic MOF-based nanoplatform involving Fenton-like reaction, Pd@MOF-525@HA, for enhanced oxygen-dependent anti-tumor therapy outcome. 
Thereinto, the porphyrinic MOF, namely MOF-525 [27], was chosen as the target support based on the following considerations: (1) The porphyrin moiety within framework can act not only as PDT and SDT agents but also as two-photon responsive unit for near infrared (NIR) light-induced PDT with deeper tissue penetration due to its large $\pi$-conjugated system and rigid planar structure [28-30]; (2) The highly dispersed Pd nanocubes can be readily encapsulated, which can react with over-expressed $\mathrm{H}_{2} \mathrm{O}_{2}$ in cancer cells to produce hydroxyl radicals $(\cdot \mathrm{OH})$ and $\mathrm{O}_{2}$ through Fenton-like reaction and then greatly alleviates the tumor hypoxia [31, 32]; (3) The hyaluronic acid (HA)-wrapping through surface modification gives rise to considerable biocompatibility and cancer cell-specific targeting ability [33-35]. The results show that the obtained two-photon responsive nanoplatform, Pd@MOF-525@HA, possess deeper tissue penetration, considerable light/ultrasonic-induced singlet oxygen $\left({ }^{1} \mathrm{O}_{2}\right)$ generation capacity, efficient oxygen generation and cancer cell specific targeting ability. Besides, we further combined two-photon fluorescence imaging to realize the combined precision anti-cancer treatment via PDT/SDT/CDT method.

\section{Results And Discussion}

The composite Pd@MOF-525 with appropriate size for cellular uptake was constructed by introducing 5,10,15,20-tetracarboxy(4-carboxyphenyl)porphyrin (TCPP), pre-synthesized $\mathbf{Z r}_{6}$ clusters and $\mathbf{P d}$ nanocubes via in-situ growth method [36]. As shown in Fig. 1A, the morphology of obtained Pd nanocubes was observed by transmission electron microscopy (TEM), which has a regular cubic structure, uniform size $(\approx 10 \mathrm{~nm})$ and good monodispersity. Moreover, the lattice spacing of $0.22 \mathrm{~nm}$ observed in the high-resolution TEM (HRTEM) image was assigned to the Pd (111) plane, confirming the structure of Pd nanocubes [37]. The TEM and scanning electron microscopy (SEM) images displayed clearly that Pd nanocubes were dispersed within MOF-525 and the size of Pd@MOF-525 increased to 130 nm (Fig. 1B-C). Simultaneously, the TEM elemental mappings were further performed for the Pd@MOF$\mathbf{5 2 5}$ composite, which proved that the homogeneous distribution of Pd element in the MOF-525 framework (Fig. 1D and Additional file 1: Fig. S1). Besides, the fabricated MOF-525 and Pd@MOF-525 was further demonstrated by the power X-ray diffraction (PXRD). These peaks could be matched with the parent framework of MOF-525 (Fig. 1E) and the characteristic peaks of Pd nanocubes were appeared at high angles of 40.0 and 46.0 (Additional file 1: Fig. S2). In addition, HA was used to further modify the surface of Pd@MOF-525 to enhance its biocompatibility and cancer-specific targeting ability for further biological applications. The zeta potential analysis (Fig. 1F) manifested a obviously change in the surface charge from a positive potential of MOF-525 (+7.16 mV) to the negative potential of Pd@MOF525 (-4.98 mV) and Pd@MOF-525@HA (-12 mV), corroborating the successful encapsulation of Pd and wrapping of HA. Moreover, as shown by dynamic light scattering (DLS) measurements (Fig. 1G), the average hydrodynamic diameters were reasonablely enlarged from $156 \mathrm{~nm}$ (MOF-525) to $173 \mathrm{~nm}$ (Pd@MOF-525@HA) and then to197 nm for Pd@MOF-525@HA. All the above data results demonstrated the successful fabrication of Pd@MOF-525@HA.

The successful preparation of Pd@MOF-525@HA inspired us to further study its photophysical properties. From the absorption spectrum of Pd@MOF-525@HA(Fig. 2A), the characteristic absorption 
for MOF-525 could be observed. First of all, considering the large $\pi$-conjugated feature of porphyrin moiety within frameworks, we investigated the reverse saturation absorption (RSA) characteristics by zscan experiments under near-infrared laser $(800 \mathrm{~nm})$ with different pulse energies (Fig. 2B) [38]. The results showed that the absorption intensity gradually enhanced with the pulse energy. Moreover, the relationship between changes of normalized $\left(\Delta T_{0}\right.$, the minimum value of $T_{N L}(Z)$ curves) transmittance with laser pulse energy (E) was fitted in the log-log scale. As illustrated in Fig. $2 \mathrm{C}$, the slope was calculated as 1.02 (the slope of the curve plus 1 is the number of effective photons absorbed) for Pd@MOF-525@HA, illustrating its two-photon absorption feature. It implies that Pd@MOF-525@HA can serve as an excellent candidate for NIR light-induced two-photon bioimaging.

Secondly, the light-induced ROS production ability of Pd@MOF-525@HA was studied systematically. As shown in Fig. 2D, the typical absorption of 9,10-anthracenediyl-bis(methylene)dimalonic acid (ABDA, ${ }^{1} \mathrm{O}_{2}$ indicator) increased under light or ultrasonic irradiation, demonstrating the excellent ${ }^{1} \mathrm{O}_{2}$ generation ability of Pd@MOF-525@HA. Notably, the well-dispersed Pd nanocubes within MOF-525 can convert $\mathrm{H}_{2} \mathrm{O}_{2}$ into $\cdot \mathrm{OH}$ and $\mathrm{O}_{2}$ through Fenton-like reaction and then further improving the ${ }^{1} \mathrm{O}_{2}$ generation ability of Pd@MOF-525@HA (Fig. 2E). Meanwhile, the oxygen production capacity of Pd@MOF-525@HA was measured by the dissolved oxygen meter to determine the concentration of $\mathrm{O}_{2}$ in the solution (Fig. $2 \mathrm{~F}$ ). It was found that the $\mathrm{O}_{2}$ concentration continued to increase after 15 minutes of repeated addition of $\mathrm{H}_{2} \mathrm{O}_{2}$, indicating that Pd@MOF-525@HA can continuously catalyze $\mathrm{H}_{2} \mathrm{O}_{2}$ to produce $\mathrm{O}_{2}$. On the other hand, the ROS fluorescent probe $2^{\prime}, 7^{\prime}$-dichlorofluorescein diacetate (DCFH-DA) is used to detect $\cdot \mathrm{OH}$ under dark condition (Fig. 2G). The emission spectrum of DCFH-DA increased significantly after adding $\mathrm{H}_{2} \mathrm{O}_{2}$, which proved its excellent catalytic capacity of $\cdot \mathrm{OH}$ production through Fenton-like reaction. Moreover, electron spin resonance trapping measurements using 5,5-dimethyl-1-pyrro-Line-N-oxide (DMPO) and 2,2,6,6tetramethylpiperidine (TEMP) as the capture agent were further carried out to verify the type of produced ROS. The typical signal of DMPO-OOH (1:2:2:1 triplet) was observed after adding $\mathrm{H}_{2} \mathrm{O}_{2}$ into Pd@MOF525@HA, indicating the production of ·OH (Fig. 2H). In addition, the characteristic signals of 4-oxoTEMPO (1:1:1 triplet) for Pd@MOF-525@HA was acquired under laser and ultrasonic irradiation, manifesting the production of ${ }^{1} \mathrm{O}_{2}$ (Fig. 2I). In this sense, Pd@MOF-525@HA provides the possibility to relieve tumor hypoxia via oxygen-generating strategy for enhanced oxygen-dependent anti-tumor therapy.

Encouraged by the two-photon feature and HA-wrapping Pd@MOF-525@HA, cellular uptake experiments with CD44 negative cells of human liver cells (QSG-7701) and CD44 positive cells of human liver cancer cell (tumor cells HepG2) were performed to evaluate its tumor cell-specific targeting and NIR light-induced fluorescence imaging ability. As shown in Fig. 3A-B, in comparison to the weak fluorescence of QSG-7701 cells incubated with Pd@MOF-525@HA under 800 nm laser irradiation, HepG2 cells group exhibited strong fluorescence. Moreover, the weak fluorescence of HepG2 cells pre-incubated with HA attribute to the preoccupation of receptor sites of CD44 cells by free HA (Additional file 1: Fig. S3) clearly demonstrated that HA-modified Pd@MOF-525 can effectively target tumor cells with CD44 receptors resulting in two-photon fluorescence imaging.Whereafter, the cytotoxicity of Pd@MOF-525@HA under 
different conditions was further researched by standard (4,5-dimethylthiazol-2-yl)-2,5-diphenyltetrazolium bromide (MTT) assay. As shown in Fig. 3C-D, the cell viability of QSG-7701 cells treated by Pd@MOF525@HA was higher than that of HepG2 cells groups with/without $\mathrm{H}_{2} \mathrm{O}_{2}$ addition demonstrating that Pd@MOF-525@HA was harmless to normal cells and the generated·OH via Fenton-like reaction could give rise to decreased cell survival rate. As revealed by Fig. 3E-H, obviously cell apoptosis could be observed upon the light and ultrasound irradiation, and the cell viability was further decreased upon $\mathrm{H}_{2} \mathrm{O}_{2}$ added due to the production of $\mathrm{O}_{2}$. Notably, in the presence of $\mathrm{H}_{2} \mathrm{O}_{2}$, the survival rate of the cells treated by light and ultrasound irradiation was only $10 \%$, which demonstrated the excellent in vitro PDT/SDT synergistic therapeutic outcome.

The in vitro PDT/SDT therapeutic effect was further evaluated through confocal laser scanning microscopy (CLSM) imaging. Firstly, the intracellular ${ }^{1} \mathrm{O}_{2}$ and $\cdot \mathrm{OH}$ generated from Pd@MOF-525@HA were detected by singlet oxygen sensor green (SOSG) and aminophenyl fluorescein (APF), respectively. As shown in Fig. 4A, obviously SOSG green fluorescence signal was collected with light/ultrasonic irradiation. Moreover, the enhanced SOSG signal from light and ultrasound worked together could be further improved when $\mathrm{H}_{2} \mathrm{O}_{2}$ added, which further unveiled that the light/ultrasonic induced ${ }^{1} \mathrm{O}_{2}$ generation ability of Pd@MOF-525@HA could be effectively boosted by $\mathrm{O}_{2}$ production from Fenton-like reaction. Simultaneously, the obvious green fluorescence of APF was observed due to the generation of $\cdot \mathrm{OH}$ (Fig. 4B) and the enhanced APF signal from $\mathrm{H}_{2} \mathrm{O}_{2}$ addition could be effectively quenched after adding $\cdot \mathrm{OH}$ scavenger (ascorbic acid, $\mathrm{AA}$ ). The above results corroborated the ROS generation ability of Pd@MOF-525@HA in cells for further treatment application. In addition, as demonstrated by the 3D fluorescence images collected by one- and two-photon fluorescence imaging of fixed mouse brain tissue (Fig. 4C-D), the deeper penetration depth (60 $\mu \mathrm{m})$ of Pd@MOF-525@HA upon 800 nm laser irradiation provided a promising platform for improved therapy outcome guided by two-photon fluorescence imaging.

Based on the deep tissue penetration and excellent intracellular ROS generation of Pd@MOF-525@HA, we deployed a thoughtful protocol to evaluate the efficacy of in vitro PDT/SDT therapy. The apoptosis was detected by using Calcein acetoxymethyl ester (Calcein AM, green) and propidium iodide ( $\mathrm{PI}, \mathrm{red})$. As shown in Fig. 5A, brighter PI signal was collected when light and ultrasound worked together than that of only light or ultrasound treatment groups. Moreover, much brighter PI signal could be observed upon $\mathrm{H}_{2} \mathrm{O}_{2}$ addition demonstrating the enhanced in vitro PDT and SDT effect by alleviating hypoxia environment. The above results were further confirmed by Annexin V-FITC/PI treatment assay (Fig. 5B). In addition, the cell apoptosis was analyzed by flow cytometry using annexin V-FITC and PI as indicators under different treatment conditions. Upon light $\left(800 \mathrm{~nm}, 100 \mathrm{~mW} \mathrm{~cm}^{-2}\right)$ and ultrasound $\left(100 \mathrm{~mW} \mathrm{~cm}^{-2}\right)$ irradiation for 15 minutes, the fraction of late apoptotic cells was $56.68 \%$, which was higher than that of only light (41.99\%) or ultrasound (30.82\%) treatment group (Fig. $5 \mathrm{C}$ ). With the addition of $\mathrm{H}_{2} \mathrm{O}_{2}$, the late apoptotic cells reached $87.73 \%$ suggesting enhanced oxygen-dependent therapeutic effect due to the generation of $\mathrm{O}_{2}$. Furthermore, HepG2 3D multicellular tumor spheroids of 3D cancer model (3D MCTs) 
were incubated with Pd@MOF-525@HA and stained with Calcein AM/PI to validate the efficiency in deep tissue. As shown in Fig. 5D, in contrast to the faint red fluorescence of control group, MCTs formed an obvious necrotic core with bright PI fluorescence upon the NIR light and ultrasound irradiation corroborating the outstanding synergistic therapeutic effect. It manifested that Pd@MOF-525@HA can be employed as a PDT and SDT combination therapy platform guided by two-photon fluorescence imaging.

\section{Conclusion}

In summary, an intelligent nanoplatform (Pd@MOF-525@HA) involving Fenton-like reaction was fabricated for enhanced oxygen-dependent anti-tumor therapy by oxygen-generating strategy. Thanks to the successful synthesis of the porphyrinic metal-organic framework, it can act not only as photo/sonosensitizers but also as two-photon responsive unit, the excellent ${ }^{1} \mathrm{O}_{2}$ generation ability leads to considerable PDT and SDT outcome with deeper tissue penetration. In addition, the therapy effect can be further enhanced due to the $\mathrm{O}_{2}$ production from Pd nanocubes through Fenton-like reaction. It provides a promising platform to relieve tumor hypoxia via oxygen-generating strategy for enhanced oxygendependent anti-tumor therapy.

\section{Materials And Methods}

\section{Materials}

All starting materials were obtained from commercial supplies and used without further purification. The chemicals of Sodium tetrachloropalladate(II) $\left(\mathrm{Na}_{2} \mathrm{PdCl}_{4}\right)$, Sodium bromide $(\mathrm{NaBr}), 2^{\prime}, 7^{\prime}-$ dichlorofluorescein diacetate (DCFH-DA), Ascorbic acid (AA) and Hyaluronic acid (HA) were purchased from Macklin Co., Ltd. The chemicals of acetone, polyvinylpyrrolidone (PVP, 58000w), N,NDimethylformamide (DMF), Ethanol and Acetic acid from Aladdin Co., Ltd. (4,5-dimethylthiazol-2-yl)-2,5diphenyltetrazolium bromide (MTT) was obtained from Beyotime Biotech Co., Ltd. (China). Calcein AM/PI Kit and Annexin V-FITC/PI Apoptosis Detection Kit was obtained from Shanghai Bestbio (China). Ultrapure water was used throughout.

\section{Synthesis Of Pd Nanocubes}

PVP (53 mg), AA (30 mg), $\mathrm{NaBr}(130 \mathrm{mg})$ were dissolved into ultrapure water (4 mL), the mixture was then stirred at $80{ }^{\circ} \mathrm{C}$ over 5 minutes. $30 \mathrm{mg}$ of $\mathrm{Na}_{2} \mathrm{PdCl}_{4}$ (dissolved in $1.5 \mathrm{~mL}$ of ultrapure water) was poured in the above mixture and stirred for 3 hours. After been cooled to room temperature, $\mathrm{Pd}$ nanocubes were collected by centrifugation and then stored it in $1 \mathrm{~mL}$ of DMF solution.

\section{Synthesis Of Pd@mof-525}


$3 \mathrm{~mL}$ of the synthesized Pd nanocubes were added to $2 \mathrm{~mL}$ DMF solution containing $100 \mathrm{mg} \mathbf{Z r}_{6}$ clusters), then the solution was stirred at room temperature for 4 hours (solution A). TCPP (50 mg) was then dissolved into DMF solution ( $5 \mathrm{~mL}$ ) and dispersed by ultrasound (solution $B$ ). Then acetic acid (6 $\mathrm{mL}$ ) was added to mixed mixture of solution A and solution B, and stirred for 12 hours. Finally, the product was collected after centrifugation and washing.

\section{Synthesis Of Pd@mof-525@ha}

HA (10 mg) was dispersed in the ultrapure water $(100 \mathrm{~mL}), 5 \mathrm{mg}$ of Pd@MOF-525 was added after ultrasonic. After stirring for 24 hours, washed with ultrapure water, and the final product was stored in ultrapure water.

\section{Apparatus}

UV-Vis absorption spectra were recorded on a UV-265 spectrophotometer. SEM was detected by REGULUS8230*. TEM was carried on a JEM-2100. PXRD patterns were recorded on SmartLab 9KW. Fluorescence measurements were performed on a Hitachi F-7000 fluorescence spectrophotometer. Onephoton and two-photon imaging data acquisition and processing were performed using Lecia TCS SP8 DIVE FALCON which equipped with single-wavelength laser and femtosecond laser (adjustable output wavelength: 680 - $1080 \mathrm{~nm}, 80 \mathrm{MHz}, 140 \mathrm{fs}$ ).

\section{Singlet Oxygen (0) Detection}

The ${ }^{1} \mathrm{O}_{2}$ was detected by 9,10-anthracenedipropanoic acid (ABDA, a singlet oxygen sensor) due to the generated ${ }^{1} \mathrm{O}_{2}$ would react with ABDA and reduce the absorbance around $378 \mathrm{~nm}$.Pd@MOF-525@HA(50 $\left.\mu \mathrm{gL}^{-1}\right), \mathrm{ABDA}(100 \mu \mathrm{M})$ and $\mathrm{H}_{2} \mathrm{O}_{2}(100 \mu \mathrm{M})$ were incubated together under white light and ultrasound $\left(1.2 \mathrm{~W} \mathrm{~cm}^{-2}\right)$ irradiation within $0-5$ minutes. The absorbance of the mixture was measured at the different time.

\section{Electron Spin Resonance (Esr) Assay}

The spin traps 2,2,6,6-tetramethylpiperidine (TEMP, trapping ${ }^{1} \mathrm{O}_{2}, 20 \mu \mathrm{L}$ ) and 5,5-dimethyl-1-pyrroline-Noxide (DMPO, trapping $\cdot \mathrm{OH}, 20 \mu \mathrm{L}$ ) were employed to detect the species of ROS generated by Pd@MOF525@HA $\left(50 \mu \mathrm{g} \mathrm{m}^{-1}\right)$. The ESR signals of the Pd@MOF-525@HA before and after LED light (range from 400 to $700 \mathrm{~nm}, 40 \mathrm{~mW} \mathrm{~cm}^{-2}$ ) and ultrasound $\left(1.2 \mathrm{~W} \mathrm{~cm}^{-2}\right)$ irradiation were recorded.

\section{Cellular Uptake Analysis}


QSG-7701 cells (CD44-negative) and HepG2 cells (CD44-positive) were seeded onto the cell culture dishes and grown to about 70\% confluency for next using. QSG-7701 cells and HepG2 cells were treated with Pd@MOF-525@HA (100 $\mu \mathrm{g} \mathrm{mL}^{-1}$, and another dish HepG2 cells were precultured with 10 times of HA before incubation with Pd@MOF-525@HA. And after 8 hours of incubation, the cellular uptake ability of Pd@MOF-525@HA were analyzed using CLSM.

\section{Cytotoxicity Assays In Cells}

The PDT/CDT/SDT effect of Pd@MOF-525@HA was studied by the methylthiazolyldiphenyltetrazolium bromide (MTT) assay. The Pd@MOF-525@HA stock solution is diluted with fresh medium to the required concentration $\left(0,30,60,90,120,150 \mu \mathrm{g} \mathrm{mL}^{-1}\right)$. Before the experiment, HepG2 cells were cultured for 24 hours in 96-well plates. Then exchange the cell culture medium with different concentrations of Pd@MOF-525@HA medium solution. They were incubated at $37^{\circ} \mathrm{C}$ for 8 hours in $5 \% \mathrm{CO}_{2}$ atmosphere, and then irradiated by laser $\left(800 \mathrm{~nm}, 1 \mathrm{~W} \mathrm{~cm}^{-2}\right)$ and ultrasound $\left(1.2 \mathrm{~W} \mathrm{~cm}^{-2}\right)$ for $15 \mathrm{~min} .100 \mu \mathrm{L}$ of fresh medium were used to exchange the cell medium solutions $20 \mu \mathrm{L}\left(5 \mathrm{mg} \mathrm{mL}^{-1}\right)$ MTT solution were added to each well following. The cell plates were then incubated for another 4 hours. After removing the MTT medium, the formazan crystals were dissolved in DMSO $\left(100 \mu \mathrm{L}\right.$ well $\left.{ }^{-1}\right)$ and the absorbance was detected at $490 \mathrm{~nm}$ using a microplate reader. And duplicated experiments have been tested.

\section{Singlet Oxygen Detection In Cells}

HepG2 cells were treated with Pd@MOF-525@HA $\left(100 \mu \mathrm{g} \mathrm{mL}^{-1}\right)$ for 8 hours, and then incubated with 1 $\mu \mathrm{M}$ singlet oxygen sensor green (SOSG) for 10 minutes. Next, HepG2 cells were washed with PBS and irradiated for 15 minutes under laser $\left(800 \mathrm{~nm}, 1 \mathrm{~W} \mathrm{~cm}^{-2}\right)$ and ultrasound $\left(1.2 \mathrm{~W} \mathrm{~cm}^{-2}\right)$. The green fluorescence was observed by CLSM with the excitation wavelength of $504 \mathrm{~nm}\left(\lambda_{\mathrm{em}}: 500-550 \mathrm{~nm}\right)$.

\section{Live/dead Assay With Calcein Am/pi}

After the HepG2 cells were washed with PBS solution twice, Pd@MOF-525@HA (100 $\left.\mu \mathrm{g} \mathrm{mL}^{-1}\right)$ was added to the above medium and incubated for 8 hours, and then the cells were treated under different conditions. Calcein AM and PI were added to detect the cells vitality of HepG2 cells. Fluorescence images are collected by CLSM.

\section{Determination Of Annexin V-fitc And Pi}

HepG2 cells were incubated with Pd@MOF-525@HA $\left(100 \mu \mathrm{g} \mathrm{mL}{ }^{-1}\right)$ at $35^{\circ} \mathrm{C}$ and $5 \% \mathrm{CO}_{2}$ for 8 hours. After adding $\mathrm{H}_{2} \mathrm{O}_{2}$, they were irradiated with laser $\left(800 \mathrm{~nm}, 1 \mathrm{~W} \mathrm{~cm}^{-2}\right)$ and ultrasound $\left(1.2 \mathrm{~W} \mathrm{~cm}^{-2}\right)$ for 15 
minutes. Then, the Annexin V-FITC $(1 \mu \mathrm{M})$ and PI $(1 \mu \mathrm{M})$ were added and incubated for 20 minutes. Fluorescence images of the cells were collected by a confocal laser scanning microscope.

\section{Flow Cytometry Study}

Cells seeded into the 6-well plates were incubated for 24 hours, the medium containing Pd@MOF525@HA $\left(100 \mu \mathrm{g} \mathrm{mL}^{-1}\right)$ was used.. After irradiated with laser $\left(800 \mathrm{~nm}, 1 \mathrm{~W} \mathrm{~cm}{ }^{-2}\right)$ and ultrasound $(1.2 \mathrm{~W}$ $\mathrm{cm}^{-2}$ ) 15 minutes, the cells were collected after centrifugation and then resuspended in binding buffer containing Propidium lodide (PI, $10 \mu \mathrm{L})$ and Annexin-V FITC $(5 \mu \mathrm{L})$ for 15 minutes in darkness. The signal was collected by a BD FACS Calibur flow cytometer (Beckaman/Gallios).

\section{The One/two-photon Fluorescence Imaging Study Of Pd@mof-525@ha}

A Lecia TCS SP8 DIVE FALCON which equipped with single-wavelength laser and femtosecond laser (adjustable output wavelength: 680 - $1080 \mathrm{~nm}, 80 \mathrm{MHz}, 140 \mathrm{fs}$ ) was employed to achieve one/twophoton fluorescence imaging. HepG2 cells were treated with Pd@MOF-525@HA for 8 hours. And then, slices were prepared from cardiac muscle tissue in Balb/c mice. The tissue sections were cut to $200 \mathrm{~mm}$ thickness. The tissue sections was incubated with Pd@MOF-525@HA for 30 minutes. The one-photon fluorescence emission was observed excitation at $458 \mathrm{~nm}\left(0.2 \mathrm{~W} / \mathrm{cm}^{2}\right)$. The two-photon fluorescence emission was observed excitation at $800 \mathrm{~nm}\left(0.2 \mathrm{~W} / \mathrm{cm}^{2}\right)$.

\section{Abbreviations}

${ }^{1} \mathrm{O}_{2}$ : singlet oxygen; PDT: photodynamic therapy; SDT: sonodynamic therapy; NIR: near infrared; ROS: reactive oxygen species; $\cdot \mathrm{OH}$ : hydroxyl radicals; RSA: reverse saturation absorption;

\section{Declarations}

\section{Acknowledgements}

We appreciate the support form National Natural Science Foundation of China (22171001 and 21701160) and Natural Science Foundation of Anhui Province of China(2108085MB49).

\section{Authors' contributions}

WenyaoDuan: Investigation, Data curation, Writing-original draft. Bo Li: Investigation, Software, Formal analysis. Wen Zhang: Investigation, Methodology.Jiaqi Li: Investigation, Methodology. Xin Yao: Investigation, Methodology. Yupeng Tian: Re- sources,Formalanalysis. JunZheng: Conceptualization, 
Methodology, Supervision. Dandan Li: Conceptualization, Methodology, Writing-review \& editing, Supervision.

\title{
Funding
}

This work was supported by a grant for the National Natural Science Foundation of China (21701160), Doctor Startup Fund (S020118002/026 and S020118002/073), Natural Science Foundation of Anhui Province of China (1908085MB30).

\section{Availability of data and materials}

All data generated or analyzed during this study are included in this publishedarticle and its additional files.

\section{Competing interests}

There are no conflicts to declare.

\section{References}

References are not available with this version

\section{Figures}

\section{Figure 1}

A TEM image of Pdnanocubes. Insert: HRTEM image of Pd nanocubes. B TEM image of Pd@MOF-525. C SEM image of Pd@MOF-525. D TEM elemental mappings of N, O, Zr and Pd of Pd@MOF-525.E PXRD patterns of simulated MOF-525, as synthesized MOF-525, Pd@MOF-525and Pd@MOF-525@HAF Zeta potential and G Hydrodynamic diameters of MOF-525, Pd@MOF-525and Pd@MOF-525@HA.

\section{Figure 2}

\begin{abstract}
A UV-Vis absorption spectra of Pd,MOF-525, Pd@MOF-525and Pd@MOF-525@HA. B Z-scan data at different pulse energy of Pd@MOF-525 under the excitation at $800 \mathrm{~nm}$. C The relationship between $\Delta \mathrm{T}_{0}$ and $E$ on a log-log scale excited by $800 \mathrm{~nm}$ laser. $D^{1} \mathrm{O}_{2}$ generation under different conditions detected by ABDA. ETime-dependent generation of ${ }^{1} \mathrm{O}_{2}$ treated with different conditions detected by ABDA.F The $\mathrm{O}_{2}$ concentration of solution treated with Pd@MOF-525@HA upon repeating addition of $\mathrm{H}_{2} \mathrm{O}_{2}$. G Detect the
\end{abstract}


production of ·OH with DCFH-DA under dark condition. H ESR signals of Pd@MOF-525@HA trapped by DMPO. IESR signals of Pd@MOF-525@HA trapped by TEMP.

\section{A Brigbtfield}

NPs

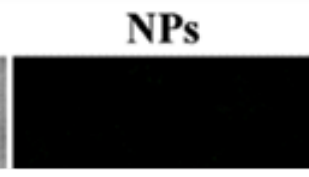

Merge

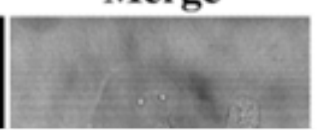

B Brigbtfield

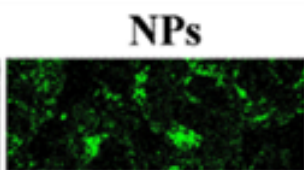

Merge

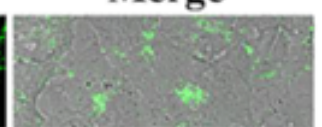

\section{Figure 3}

A Confocal images to verify the cellular uptake ofPd@MOF-525@HA $(100 \mu \mathrm{g} / \mathrm{mL})$ incubated with QSG7701 cells. B Confocal images to verify the cellular uptake ofPd@MOF-525@HA (100 $\mu \mathrm{g} / \mathrm{mL})$ incubated withHepG2 cells. CCell viability of QSG-7701 cells incubated with Pd@MOF-525@HA.D-H Cell viability of HepG2 cells incubated with Pd@MOF-525@HA under different treatments (Laser: 800 nm, 1W cm ${ }^{-2}$; US: $\left.1.2 \mathrm{~W} \mathrm{~cm}^{-2} ; \mathrm{H}_{2} \mathrm{O}_{2}: 100 \mu \mathrm{M}\right)$. 


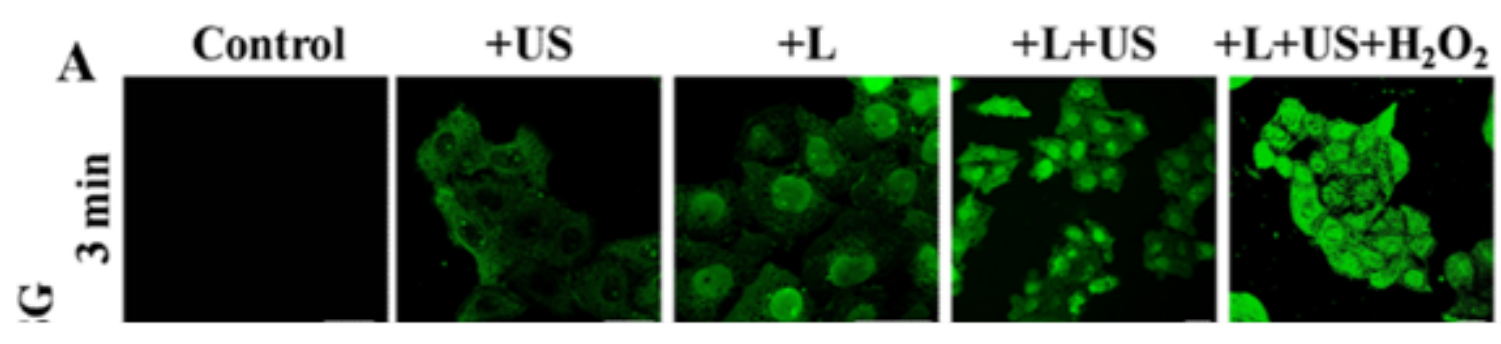

\section{Figure 4}

A CLSM images of HepG2 cells treated with Pd@MOF-525@HA and detect the generation of ${ }^{1} \mathrm{O}_{2}$ using SOSG (Laser: $800 \mathrm{~nm}, 1 \mathrm{~W} \mathrm{~cm}{ }^{-2}$; US: $1.2 \mathrm{~W} \mathrm{~cm}^{-2} ; \mathrm{H}_{2} \mathrm{O}_{2}: 100 \mu \mathrm{M}$; Scale bar: $25 \mu \mathrm{m}$ ). B CLSM images of HepG2 cells treated with Pd@MOF-525@HA and detect the generation of ·OH using APF (ascorbic acid: an inhibitor with $\cdot \mathrm{OH}, \mathrm{AA}: 25 \mu \mathrm{M} ; \mathrm{H}_{2} \mathrm{O}_{2}: 100 \mu \mathrm{M}$; scale bar: $\left.25 \mu \mathrm{m}\right)$. COne-photon (458 nm, $0.2 \mathrm{~W} \mathrm{~cm}^{-2}$ ) and D Two-photon (800 nm, $\left.0.2 \mathrm{~W} \mathrm{~cm}^{-2}\right) 3 \mathrm{D}$ fluorescence images of tissue section.

\section{Figure 5}

A CLSM images of HepG2 cells treated with Pd@MOF-525@HA after different treatment followed by stained with Calcein AM/PI (Laser: $800 \mathrm{~nm}, 1 \mathrm{~W} \mathrm{~cm}{ }^{-2}$; US: $1.2 \mathrm{~W} \mathrm{~cm}^{-2} ; \mathrm{H}_{2} \mathrm{O}_{2}: 100 \mu \mathrm{M}$; Scale bar: $100 \mu \mathrm{m}$ ). B CLSM images of HepG2 cells incubated with Pd@MOF-525@HA for 12hours (Annexin V-FITC/PI were indicators of apoptosis, scale bar: $25 \mu \mathrm{m}$ ).CCell apoptosis assays of HepG2 cells treated with Pd@MOF- 
525@HA after different treatment followed by stained with annexin V-FITC and PI.D3D fluorescence images of MCTsafter different treatments (Laser: $800 \mathrm{~nm}, 1 \mathrm{~W} \mathrm{~cm}-2$; US: $1.2 \mathrm{~W} \mathrm{~cm}^{-2} ; \mathrm{H}_{2} \mathrm{O}_{2}: 100 \mu \mathrm{M}$ ).

\section{Supplementary Files}

This is a list of supplementary files associated with this preprint. Click to download.

- GraphicalAbstract.docx

- Sl.docx 